\title{
Dominant Color and Texture Approached for Content Based Video Images Retrieval
}

\author{
Ranjit.M.Shende ${ }^{1}$, Dr P.N.Chatur ${ }^{2}$ \\ ${ }^{I}$ Department of Computer Science and Engineering, Government College of Engineering, Amravati \\ (Maharashtra), 444606, India. \\ ${ }^{2}$ Department of Computer Science and Engineering, Government College of Engineering, Amravati \\ (Maharashtra), 444606, India.
}

\begin{abstract}
Content-based retrieval allows finding information by searching its content rather than its attributes. Content-based search and retrieval of video data becomes a challenging and important problem. Every year video content is growing in volume and there are different techniques available to capture, compress, display, store and transmit video while editing and manipulating video based on their content is still a non-trivial activity. Recent advances in multimedia technologies allow the capture and storage of video data with relatively inexpensive computers. However, without appropriate search techniques all these data are hardly usable. Today research is focused on video retrieval.Moreover, content-based video retrieval system requires first of all segment the video stream into separate shots. Video Shot Afterwards features are extracted for video shots representation. And finally, choose a similarity/distance metric and an algorithm that is efficient enough to retrieve query - related videos results. There are two main issues in this process; the first is how to determine the best way for video segmentation and key frame selection. The second is the features used for video representation. Various features can be extracted for this sake including either low or high level features. A key issue is how to bridge the gap between low and high level features. In this paper we presented approach for content based video retrieval based on Dominant color and texture of a video image. We also talk about video Representation, feature extraction from like texture, dominant color and color histogram from video frame.

Keywords- Video retrieval, dominant color, Gray level co occurrence matrix. Feature extraction, Key frame extraction, Video representation, and Video segmentation. Image Retrieval, color Histogram
\end{abstract}

\section{Introduction}

Content based video retrieval system has general two stages The first one, the database population stage, performs the following tasks: Video segmentation: Segment the video into constituent shots, Key frame selection: Select one frame or more to represent each shot and Feature extraction: Extract low-level and other features from key frames or their interrelationships in order to represent these frames, hence shots. The second stage, the retrieval subsystem processes the presented query performs similarity matching operations, and finally displays results to the user. [1].

Retrieval of multimedia data is mainly divided into two parts [23].

- Text Based Retrieval

- Content Based Retrieval

Text retrieval is an easy process and it has been used for long time. But it has so many problems like manual text annotations, selecting a text for process. In content based retrieval spatial features like color, shape, texture and the temporal features like motion can be used to retrieve videos.

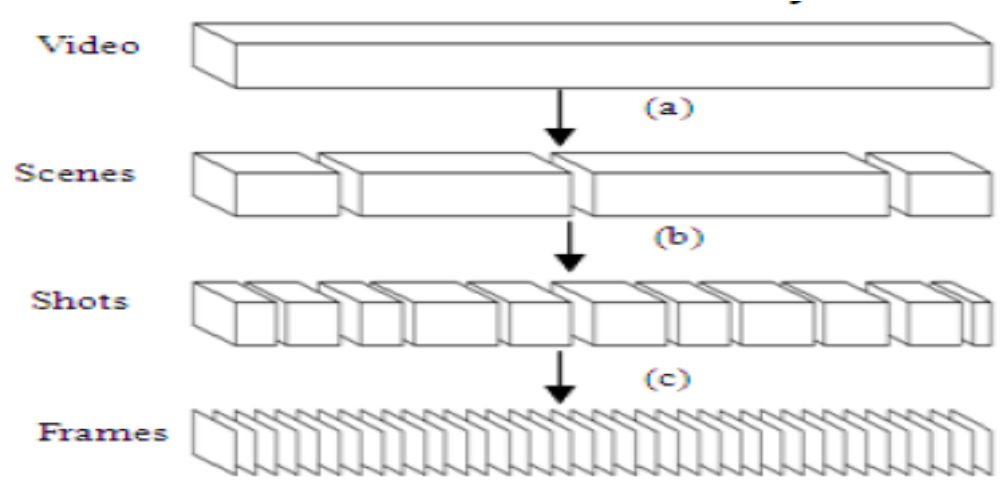

Figure 1: (a) Video into scenes (b) Scenes into shots

(c) Shots into Frames 
Video processing always is performed on frames which are basic block of video. Group of frames captured together is called shot. Few minutes shot may contain hundreds of frames, which makes video large in size. Storing and processing theses individual frames are memory and computational expensive. [5]. The Internet forms today's largest source of Information containing a high density of multimedia objects and its content is often semantically related. The identification of relevant media objects in such a vast collection poses a major problem that is studied in the area of multimedia information retrieval. Before the emergence of content-based retrieval, media was annotated with text, allowing the media to be accessed by text-based searching based on the classification of subject or semantics. In typical content-based retrieval systems, the contents of the media in the database are extracted and described by multi-dimensional feature vectors, also called descriptors. [3] Video processing always is performed on frames which are basic block of video. Group of frames captured together is called shot. Few minutes shot may contain hundreds of frames, which makes video large in size. Storing and processing theses individual frames are memory and computational expensive [6]. Content based video retrieval is a collection of videos. To begin with, the system splits the video into a sequence of elementary shots and extracts a small number of representative frames from each shot and subsequently calculates frame descriptors depending on the Motion, Edge, Color and Texture features. The motion, edge histogram, color histogram and texture features of the elementary video shots are extracted by employing Fast Fourier transform and L2 norm distance function, Statistical approach The elementary video shots features, extracted using the above approaches, are stored in feature library The color, edge, texture and motion features are extracted for a query video clip and evaluated against the features in the feature library[16].Each video is divided in to scenes and each scene consists of meaning full shots. A shot is defined as one or more frames. Shot represents a continuous action in time or space. A frame has a real world objects in it [2].

\section{Video Reprayzentation}

Video sequence contain large amount of information. Video segmentation is first step towards the content based video search aiming to segment moving objects in video sequences [1]. Video sequences are rich in information, large in storage requirements, and have a time dimension. Thus it is extremely useful to have effective video representation and abstraction tools so that video content can be represented compactly.[5] Compact representation of video allows the user to see the main video contents quickly without going through the video sequentially. Fig. 2 shows video is divided into no of shot, and shot contain number of frame.

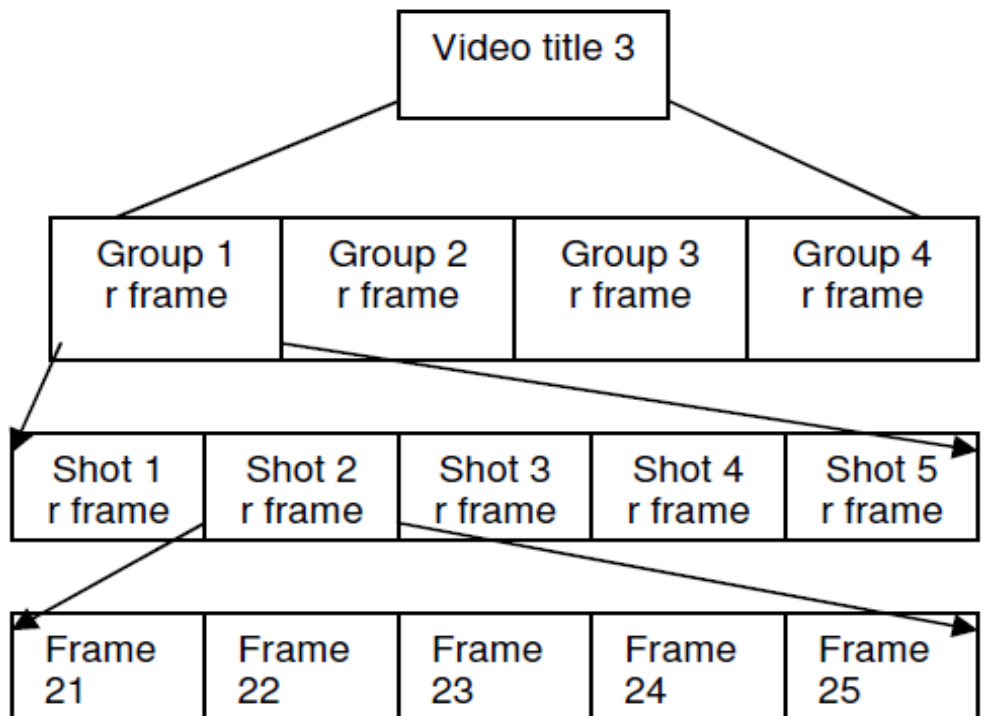

Figure 2: Video Representation

\section{Proposed Method}

Only simple features of image information cannot get comprehensive description of image content. We consider the color and texture features combining not only be able to express more image information, but also to describe image from the different aspects for more detailed information in order to obtain better search results. The proposed method is based on dominant color and texture features and Histogram of an image. Video Retrieval algorithm is as follows: 


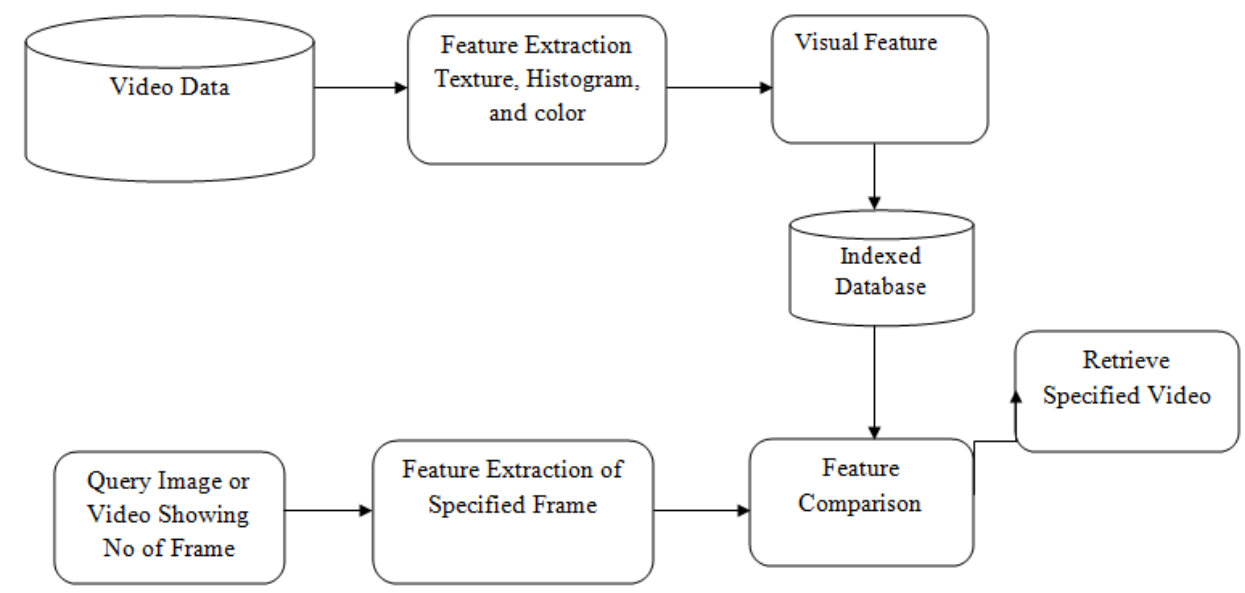

Figure 3: Block Diagram of Proposed Video Retrieval System

Step 1: First divide the video into no of image or frame

Step2: Uniformly divide each image in the database and the target image into 16-coarse partitions as shown in Fig.1.

Step3: For each partition, the Centroid of each partition is selected as its dominant color.

Step4: Obtain texture features (Energy, Contrast, Entropy and inverse difference) Gray Level Co-Occurrence Matrix (GLCM).

Step 5: Extract Feature from colour histogram for Efficient Video retrieval

Step5: construct a combined feature vector for color and texture and Color Histogram

Step6: Find the distances between feature vector of query Video and the feature vectors of target Video using weighted and normalized Euclidean distance.

Step7: Sort the Euclidean distances by comparing the query video and database video

Step8: Retrieve most similar Video with minimum Distance from Video data.

\subsection{Color Feature Representation}

\section{Feature Extractions}

In general, color is one of the most dominant and distinguishable low-level visual features in describing video image.[7] Many CBIR systems employ color to retrieve images In theory, it will lead to minimum error by extracting color feature for retrieval using real color image directly, but the problem is that the computation cost and storage required will expand rapidly In fact, for a given color video image, the number of actual colors only occupies a small proportion of the total number of colors in the whole color space, and further observation shows that some dominant colors cover a majority of pixels[8]

\subsection{Extraction of dominant color of an image:}

The procedure to extract dominant color of an image is as follows: According to number of experiments, the selection of color space is not a critical issue for Dominant colour descriptor extraction. Therefore, for simplicity and without loss of generality, the RGB colour space is used[17].Firstly, the RGB color space is uniformly divided into 8 coarse partitions, as shown in Fig. 1 colors located on the same partitioned block, they are assumed to be similar. After the above coarse partition, the centroid of each partition ("color Bin" in MPEG-7) is selected as its quantized color.

Let $\mathrm{X}=(\mathrm{XR}, \mathrm{XG}, \mathrm{XB})$ represent color components of a pixel with color components Red, Green, and Blue, and $\mathrm{Ci}$ be the quantized color for partition i $[17,18]$ The average value of color distribution for each partition center can be calculated by

$$
\overline{\mathrm{X}}_{\mathrm{i}}=\frac{\sum_{\mathrm{X} \in \mathrm{ci}} \mathrm{X}}{\sum_{\mathrm{X} \in \mathrm{ci}} 1}
$$

After the average values are obtained, each quantized color can be determined by using

$$
\mathrm{C}_{\mathrm{i}=}\left(\overline{X i}^{R}, \overline{X i}^{G}, \overline{X i}^{B}\right)(1 \leq i \leq 8)
$$

In this way, the dominant colors of a video image will be obtained. 


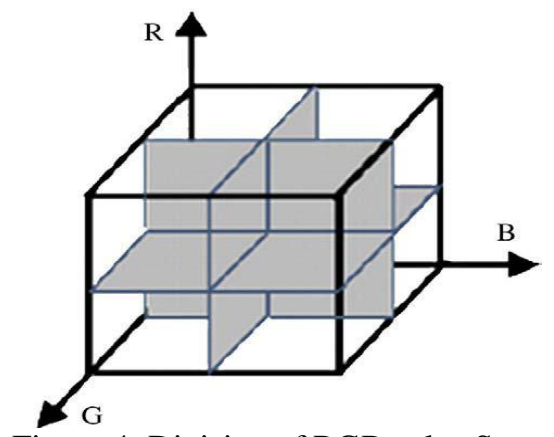

Figure 4: Division of RGB color Space

\subsection{Texture feature}

Texture feature extraction based on GLCM:

GLCM creates a matrix with the directions and distances between pixels, and then extracts meaningful statistics from the matrix as texture features. In this paper, we propose a texture representation for image retrieval based on GLCM. GLCM $[16,17]$ is created in four directions with the distance between pixels as one. Texture features are extracted from the statistics of this matrix. Four GLCM texture features are commonly used which are in following manner:

$$
P(i, j \mid d, \theta)=\frac{P(i, j \mid d, \theta)}{\sum_{i} \sum_{j} P(i, j \mid d, \theta)}
$$

GLCM $[17,18]$ is composed of the probability value, it is defined by P $(i, j \mid d, \theta)$, GLCM expresses the texture feature according the correlation of the couple pixels gray-level value at different positions. It quantification ally describes the texture feature. In this paper, four texture features are considered. [14] They include energy, contrast, entropy, inverse difference.

$$
\text { Energy } \mathrm{E}=\sum_{x} \Sigma_{y} P(x, y)^{2}
$$

It is a texture measure of gray-scale image represents homogeneity changing, reflecting the distribution of image gray-scale uniformity of weight and texture. Contrast is the main diagonal near the moment of inertia, which measures how the values of the matrix are distributed and number of images of local changes reflecting the image clarity and texture of shadow depth. Large Contrast represents deeper texture.

$$
\text { Contrast } \mathrm{I}=\sum \sum(\mathrm{x}-\mathrm{y})^{2} \mathrm{P}(\mathrm{x}, \mathrm{y})
$$

Contrast is the main diagonal near the moment of inertia, which measure the value of the matrix is distributed and images of local changes in number, reflecting the image clarity and texture of shadow depth. Contrast is large means texture is deeper.

Entropy $\mathrm{S}=-\sum_{x} \sum_{y} p(x, y) \log p(x, y)$

Entropy measures randomness in the image texture. Entropy is minimum when the co-occurrence matrix for all values is equal. On the other hand, if the value of co-occurrence matrix is very uneven, its value is greater. Therefore, the maximum entropy implied by the image gray distribution is random.

$$
\text { Inverse Difference } \mathrm{H}=\sum_{x} \sum_{y} \frac{1}{1+(x-y)^{2}} p(x, y)
$$

It measures number of local changes in image texture. Its value in large is illustrated that image texture between the different regions of the lack of change and partial very evenly. Here $\mathrm{p}(\mathrm{x}, \mathrm{y})$ is the gray-level value at the Coordinate (x, y).

The texture features are computed for an image when $\mathrm{d}=1$ and $=0^{\circ}, 45^{\circ}, 90^{\circ}, 135^{\circ}$.In each direction four texture features are calculated. [17] They are used as texture feature descriptor. Combined feature vector of Color and texture, Histogram is formulated. 
Fig.4 shows the feature extraction from feature like dominant color, texture and histogram of an image, which are stored in xls db file. Gray level concurrence matrix returns four values Energy, Contrast, Entropy and Inverse Difference. After getting all feature extract stored in database and will be use as similarity measure between query video feature and feature stored in xls file.

\begin{tabular}{|c|c|c|c|c|c|c|c|c|c|c|c|c|c|c|c|c|c|c|c|c|}
\hline 1 & IA & BB & IC & ID & IE & If & IG & $\mathbb{I H}$ & ॥ & IJ & IK & IL & IM & IN & 10 & IP & IQ & $\mathbb{R}$ & IS & IT \\
\hline 60 & 422 & 445 & 416 & 317 & 390 & 377 & 406 & 460 & 526 & 692 & 519 & 524 & 478 & 398 & 401 & 279 & 433 & 238 & 2 & 121 \\
\hline 61 & 419 & 479 & 435 & 335 & 364 & 365 & 456 & 489 & 580 & 626 & 548 & 486 & 532 & 394 & 371 & 275 & 422 & 240 & 2 & 126 \\
\hline 62 & 428 & 572 & 1064 & 841 & 581 & 376 & 317 & 308 & 184 & 172 & 200 & 151 & 221 & 222 & 193 & 667 & 455 & 303 & 2 & 131 \\
\hline 63 & 2 & 0 & 0 & 1 & 1 & 4 & 4 & 9 & 12 & 8 & 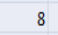 & 13 & 5 & 2 & 48 & 92 & 12 & 6 & 2 & 136 \\
\hline 64 & 2020 & 1282 & 1353 & 987 & 727 & 626 & 681 & 518 & 507 & 598 & 441 & 392 & 307 & 361 & 348 & 320 & 366 & 318 & 2 & 141 \\
\hline 65 & 266 & 11 & 0 & 0 & 0 & 0 & 3 & 5 & 7 & 6 & 20 & 10 & 7 & 4 & 38 & 18 & 16 & 24 & 2 & 146 \\
\hline 66 & 245 & 327 & 225 & 261 & 228 & 158 & 296 & 267 & 203 & 110 & 52 & 48 & 48 & 58 & 70 & 95 & 133 & 113 & 2 & 151 \\
\hline 67 & 28 & 48 & 27 & 2 & 1 & 4 & 9 & 12 & 12 & 7 & 12 & 3 & 2 & 8 & 72 & 36 & 42 & 32 & 2 & 156 \\
\hline 68 & 75 & 31 & 3 & 4 & 9 & 11 & 7 & 12 & 8 & 12 & 7 & 7 & 5 & 6 & 19 & 35 & 36 & 30 & 2 & 161 \\
\hline 69 & 2570 & 2476 & 2612 & 2561 & 2594 & 2882 & 2781 & 2954 & 2825 & 2739 & 2844 & 2986 & 3100 & 3309 & 4000 & 3386 & 3029 & 3066 & 3 & 1 \\
\hline 70 & 3293 & 3144 & 3115 & 2954 & 2946 & 3061 & 3002 & 2688 & 2562 & 2577 & 2464 & 2595 & 2829 & 2812 & 2886 & 2858 & 2117 & 1924 & 3 & 11 \\
\hline 71 & 1451 & 1375 & 1302 & 1468 & 1228 & 1219 & 1389 & 1191 & 1196 & 1271 & 1136 & 1165 & 1116 & 1054 & 974 & 902 & 926 & 791 & 3 & 21 \\
\hline 72 & 2092 & 2071 & 2096 & 2154 & 2251 & 2189 & 2137 & 2269 & 2447 & 2494 & 2389 & 2337 & 1915 & 1588 & 1460 & 1283 & 1186 & 1053 & 3 & 31 \\
\hline 73 & 2562 & 2544 & 2461 & 2363 & 2451 & 2369 & 2362 & 2438 & 2325 & 2239 & 2266 & 2198 & 2105 & 2163 & 2182 & 2461 & 2478 & 2489 & 3 & 41 \\
\hline 74 & 1962 & 1842 & 1895 & 1825 & 1897 & 1851 & 1893 & 1870 & 1894 & 1930 & 1996 & 1935 & 1981 & 2011 & 2122 & 2128 & 2215 & 2190 & 3 & 51 \\
\hline 75 & 1758 & 1831 & 1890 & 1847 & 1769 & 1747 & 1716 & 1780 & 1962 & 1957 & 1844 & 1878 & 1833 & 1983 & 1900 & 2101 & 2159 & 2237 & 3 & 61 \\
\hline 76 & 2751 & 2681 & 2610 & 2548 & 2498 & 2585 & 2614 & 2452 & 2496 & 2581 & 2453 & 2576 & 2572 & 2613 & 2721 & 2657 & 2685 & 2720 & 3 & 71 \\
\hline 77 & 729 & 721 & 704 & 746 & 726 & 756 & 777 & 661 & 711 & 676 & 587 & 563 & 567 & 545 & 573 & 498 & 505 & 492 & 3 & 81 \\
\hline 78 & 3225 & 2787 & 3292 & 3239 & 2551 & 4061 & 3961 & 3100 & 4516 & 2569 & 2803 & 3933 & 4367 & 5285 & 3288 & 3910 & 2654 & 4554 & 3 & 91 \\
\hline 79 & 3098 & 3088 & 3104 & 3356 & 2945 & 2968 & 3203 & 3099 & 3080 & 3049 & 3341 & 3088 & 3084 & 3298 & 3327 & 3506 & 3602 & 3822 & 3 & 101 \\
\hline 80 & 1537 & 1537 & 1677 & 1566 & 1658 & 1600 & 1587 & 1562 & 1587 & 1564 & 1538 & 1559 & 1664 & 1594 & 1635 & 1614 & 1652 & 1765 & 3 & 111 \\
\hline 81 & 728 & 781 & 715 & 770 & 762 & 749 & 675 & 610 & 654 & 600 & 603 & 597 & 571 & 541 & 572 & 588 & 573 & 531 & 3 & 121 \\
\hline 82 & 2823 & 2864 & 3118 & 2930 & 2821 & 3082 & 2821 & 3224 & 3040 & 3812 & 2980 & 3349 & 3511 & 3164 & 3352 & 3970 & 3546 & 3680 & 3 & 131 \\
\hline 83 & 2157 & 2403 & 2490 & 2464 & 2411 & 2303 & 2185 & 2113 & 1954 & 1946 & 1815 & 1693 & 1624 & 1578 & 1590 & 1483 & 1438 & 1449 & 3 & 141 \\
\hline 84 & 1383 & 1336 & 1403 & 1396 & 1389 & 1276 & 1376 & 1348 & 1373 & 1439 & 1359 & 1334 & 1326 & 1298 & 1367 & 1413 & 1357 & 1336 & 3 & 151 \\
\hline
\end{tabular}

Figure 4: Feature Extractions from Video Frame

\section{Conclusion}

Content-based retrieval of visual information is an emerging area of research which has been in limelight amongst the researchers and experimenters. Video retrieval system is useful for video editing, video archiving, and interactive video and multimedia information systems. This paper presented the video representation in terms of shot and shot is divided into number of frame, feature selection, Extraction of feature like texture, color and Histogram of video image the proposed content based video retrieval system. We also provide screen shot of features calculated from texture, color, and histogram of video image using Matlab.

\section{Reference}

[1] Shweta Ghodeswar, B.B.Meshram Technicians "Content Based Video Retrieval"

[2] R. Kanagavalli, Dr. K. Duraiswamy “Object Based Video Retrievals” International Journal of Communications and Engineering Volume 06- No.6, Issue: 01 March2012.

[3] Dr. S. D. Sawarkar, V. S. Kubde "Content Based Video Retrieval using trajectory andVelocity features" International Journal of Electronics and Computer Science Engineering ISSN- 2277-1956

[4] Ali Amiri, Mahmood Fathy, and Atusa Naseri "A Novel Video Retrieval System Using GED-based Similarity Measure" International Journal of Signal Processing, Image Processing and Pattern Recognition Vol. 2, No.3, September 2009

[5] S.Thanga Ramya 1 P.Rangarajan "Knowledge Based Methods for Video Data Retrieval” International Journal of Computer Science \& Information Technology (IJCSIT) Vol 3, No 5, Oct 2011

[6] B. V. Patel A. V. Deorankar, B. B. Meshram "Content Based Video Retrieval using Entropy, Edge Detection, Black and White Color Features" Computer Engineering and Technology (ICCET), 2010 2nd International Conference on

[7] Gao, X. and X. Tang, 2002. Unsupervised video shot segmentation and model-free anchorperson detection for news video story parsing. IEEE Trans. Circuits Syst. Video Technol., 12: 765-776.

[8] Shan Li, Moon-Chuen Lee, 2005. An improved sliding window method for shot change detection. Proceeding of the 7th IASTED International Conference Signal and Image Processing, Aug. 15-17, IIonolulu, IIawaii, USA, pp: 464-468.

[9] Hamdy K. Elminir, Mohamed Abu ElSoud "Multi feature content based video retrieval using high level semantic concept" IJCSI International Journal of Computer Science Issues, Vol. 9, Issue 4, No 2, July 2012

[10] John, S., Boreczky and D. Lynn, 1998. "A hidden Markova model framework for video segmentation using audio an image features". In: Proceedings of IEEE International conference on Acoustics, Speech and Signal Processing, May 12-15, 6:

[11] Weiming Hu, Niangua Xie, Li, Xianglin Zeng, and Stephen Mayban" Survey on Visual Content-Based Video Indexing and Retrieval" IEEE Transaction on System, Man, and cybernetics — part C: application and Reviews, Vol. 41, no. 6, November 2011

[12] P. Geetha and Vasumathi Narayanan" A Survey of Content-Based Video Retrieval" Journal of Computer Science 4 (6): 474-486, 2008 ISSN 1549-3636

[13] M.Babu Rao Dr.B.Prabhakara Rao Dr.A.Govardhan "Content Based Image Retrieval using Dominant Colour and Texture Features" International Journal of Computer science and information security Vol. 9,No.2 2011

[14] Xiang-Yang Wang, Yong-Jian Yu, Hong-Ying Yang "An effective image retrieval scheme using color, texture and shape features "Computer Standards and Interface Science Direct 
[15] T.N.Shanmugam and Priya Rajendran “An Enhance Content based video retrieval system based on query clip” International Journal of Research and Reviews in Applied Sciences ISSN: 2076-734X, EISSN: 2076-7366 Volume 1, Issue 3

[16] Fan Hui-Kong "Image Retrieval using Both Colour and texture features " Proceedings of the Eighth International Conference on Machine Learning and Cybernetics, Baoding, 12-15 July 2009

[17] M.Babu Rao R.B.Prabhakara Rao Dr.A.Govardhan "Content based image retrieval using dominant colour and texture feature" International Journal of Computer Science and Information Security, Vol. 9, No. 2, February 2011

[18] C V. Jawahar, BalaKrishna Chennupati, Balamanohar Paluri and Nataraj Jammalamadaka, "Video Retrieval Based on Textual Queries", in Proceedings of the Thirteenth International Conference on Advanced Computing and Communications, Coimbatore, December 2005.

[19] O'Toole, C., A. Smeaton, N. Murphy and S. Marlow, 1999. Evaluation of automatic shot boundary detection on a large video suite. In: $2^{\text {nd }}$ U.K. Conference Image Retrieval: The Challengef Image Retrieval, Feb. 25-26, Newcastle, U.K.

[20] C.-W. Ngo, H.-J. Zhang and T.-C. Pong, "Recent advance In Content based video analysis," International Journal of Image and Graphics, vol.1, no. 3, pp. 445-468, 2001.

[21] Yi Wu, Y. Zhuang, and Y. Pan: "Content-Based Video Similarity Model," In Proc. of the 8th ACM Int. Multimedia Conf. on Multimedia, USA,

[22] Anil Jain, Aditya Vailaya, Wei Xiong., "Query by video Clip," In Proceedings of Fourteenth International Conference on Pattern Recognition, vol.1. pp: 909-911, 16-20 Aug 1998.

[23] Jang-Hui Kim, Hye-Youn Lim, and Dae-Seog Kang,"An Implementation of the Video Retrieval System by Video Segmentation".Proceedingsof APCC 2008. 